\title{
ANATOMIA ECOLÓGICA DA MADEIRA DE RUDGEA VIBURNOIDES (CHAM.) BENTH. EM CAMPO CERRADO E RUPESTRE ${ }^{1}$
}

\author{
JOÃO CARLOS FERREIRA DE MELO JÚNIOR², MAIARA MATILDE DA SILVA ${ }^{3}$ \\ PATRÍCIA SOFFIATTI ${ }^{4}$
}

\begin{abstract}
RESUMO
O presente estudo compara a anatomia do lenho de Rudgea viburnoides em duas fisionomias de Cerrado. O grupo amostral foi composto de cinco espécimes em cada fisionomia (campo cerrado e campo rupestre). Preparações histológicas e caracterização anatômica seguiram os procedimentos usuais em anatomia da madeira. Ocorreram diferenças estatisticamente significativas entre as populações para atributos de vasos, fibras e parênquima radial. Cristais prismáticos foram exclusivos no lenho em campo rupestre e tilos em campo cerrado. Em ambas as fisionomias foram encontradas máculas no lenho. O índice de mesomorfia evidenciou que a espécie está adaptada a ambientes secos, enquanto os índices de condutividade e vulnerabilidade mostraram que os espécimes de campo rupestre são mais eficientes na condução hidráulica, porém mais propensos à cavitação. As características anatômicas verificadas no lenho evidenciam variações intraespecíficas distintivas entre as populações estudadas e podem ser interpretadas como respostas adaptativas da espécie à heterogeneidade ambiente das fisionomias de cerrado.
\end{abstract}

Palavras-chave: anatomia funcional, anatomia do lenho, savana

\section{ABSTRACT}

[Ecological wood anatomy of Rudgea viburnoides (Cham.) Benth. (Rubiaceae) in two Cerrado physiognomies (closed field and rock field)].

This study compares the wood anatomy of Rudgea viburnoides in two Cerrado physiognomies. The sample group was composed of five specimens in each physiognomy (closed field and rock field). Histological preparations and anatomical characterization followed the usual procedures in wood anatomy. Statistically significant differences occurred between populations for attributes of vessels, fibers and radial parenchyma. Prismatic crystals were unique in the wood on rock field and tyloses in closed field physiognomies. On both faces stains were found in the wood. The mesomorphic index showed that the species is adapted to dry environments, while the vulnerability index and conductivity have shown that specimens from rock field are more effective in hydraulic driving, but more prone to cavitation. The anatomical features observed in the wood show distinctive intraspecific variation among the populations studied and can be interpreted as adaptive responses of species to environmental heterogeneity of cerrado physiognomies.

Keywords: functional anatomy, wood anatomy, savana

1 Recebido em 09-7-2016 e aceito para publicação em 15-9-2016.

2 Biólogo, Doutor, Professor Titular do Departamento de Ciências Biológicas, Laboratório de Anatomia e Ecologia Vegetal, Universidade da Região de Joinville, Joinville, Santa Catarina, Brasil. joao.melo@ univille.br

Bióloga, Mestranda, Programa de Pós-Graduação em Ecologia e Conservação, Universidade Federal do Paraná, SCB, Departamento de Botânica, Caixa Postal 19031, CEP 81531-990, Curitiba, Paraná, Brasil. maaiara27@gmail.com

4 Bióloga, Doutora, Professora do Departamento de Botânica, Universidade Federal do Paraná, SCB, Departamento de Botânica, Caixa Postal 19031, CEP 81531990, Curitiba, Brasil. Psoffiatti.ufpr@gmail.com

\section{INTRODUÇÃO E REVISÃO DE LITERA-} TURA

Considerado o segundo maior bioma brasileiro, o Cerrado ocupa $21 \%$ do território nacional. Cerca de 55\% da área do Cerrado já foi desmatada ou transformada pela ação humana, o que equivale a uma área de $880.000 \mathrm{~km}^{2}$ (Klink $\&$ Machado, 2005). Abriga uma expressiva diversidade biológica, caracterizada pela ocorrência de aproximadamente 7000 espécies de Angiospermas, cujas famílias Fabaceae, Vochysiaceae, Annonaceae, Bignoniaceae, 
Myrtaceae e Rubiaceae são tidas como detentoras de grande riqueza (Shepherd, 2000). A espécie arbórea Rudgea viburnoides (Cham.) Benth., pertencente à família Rubiaceae, é conhecida popularmente por congonha, congonhade-bugre, bugre e cotó-cotó, e apresenta ampla distribuição no cerrado (Alves, 2004; Durigan et al. 2004), podendo ser observada desde a fisionomia de campo sujo até campo rupestre. Esse aspecto torna a espécie interessante a estudos ecológicos e funcionais.

A anatomia ecológica do lenho abrange o estudo das influências ambientais sobre as variações estruturais de espécies lenhosas, sendo conhecida como a abordagem ecológica de anatomia da madeira (Baas, 1973). Estudos de anatomia de lenho têm sido realizados no sentido de relacionar as características anatômicas dos vegetais aos diferentes mecanismos de colonização, estabelecimento e desenvolvimento de espécies em seu ambiente natural, assim como as consequiências advindas das modificações desses ambientes (Marques et al., 2012). Desta forma, diferentes condições ambientais podem produzir distintos fenótipos em plantas, sendo tal resposta denominada plasticidade fenotípica (Bradshaw, 2006). As relações entre a estrutura da madeira e as características climáticas têm sido amplamente estudadas, sendo Baas (1973) e Carlquist (1975) os pioneiros nesses estudos. Nesta perspectiva, uma vasta literatura tem sido produzida de forma mais ampla sobre a flora brasileira (Chimello; Mattos-Filho, 1988; Alves; Angyalossy-Alfonso, 2000; Alves; AngyalossyAlfonso, 2002; Barros et al., 2006), ou mais focadas em certos biomas, como o cerrado (Ceccantini, 1996; Machado et al., 1997; Marcati et al., 2001; Machado et al., 2007; Voigt et al., 2010; Melo Jr. et al., 2012; Sonsin, 2014).

Dentre os estudos anatômicos realizados com Rubiaceae, destacam-se os de natureza descritiva de espécies da América do Sul (KoekNoorman, 1972; Koek-Noorman; Hogeweg, 1974; Zevallos Pollito; Tomazello, 2006; Patel; Patel, 2011; Siegloch et al., 2011; Williams; Leon, 2011; Sonsin, 2014) e da América do
Norte (Rogers, 2005). Sobre Rudgea viburnoides, a literatura dispõe apenas de um trabalho direcionado à sua caracterização química e anatômica, numa perspectiva fitoquímica (Alves et al., 2004).

Considerando a escassez de pesquisas com enfoque ecológico sobre o lenho de espécies de Rubiaceae do cerrado brasileiro, e face à grande diversidade de plantas registradas para esse bioma, o presente estudo objetiva comparar a anatomia do lenho de Rudgea viburnoides em duas fisionomias do cerrado, partindo-se do pressuposto de que as diferentes condições ambientais influenciam a estrutura da madeira, de forma a otimizar a relação eficiência x segurança no transporte hídrico.

\section{MATERIAL E MÉTODOS}

As áreas de estudo compreendem duas fisionomias de cerrado do Brasil central, localizadas na porção Norte do estado de Minas Gerais (Figura 1A). Tais fisionomias são assim caracterizadas:

a) campo cerrado: fisionomia localizada no município de Matozinhos, nas coordenadas geográficas $19^{\circ} 27^{\prime} \mathrm{S}$ e $43^{\circ} 58^{\prime} \mathrm{W}$ e situada a $711 \mathrm{~m}$ de altitude. Apresenta temperatura média anual de $22^{\circ} \mathrm{C}$ e precipitação média anual entre 900 e $1300 \mathrm{~mm}$. O solo é classificado como latossolo vermelho, sendo tipicamente profundo, álico e pobre em nutrientes (Embrapa, 2013) (Figura 1B).

b) campo rupestre: fisionomia situada na Serra do Cipó, a qual compõe a região montanhosa da porção sul da Cadeia do Espinhaço, sob as coordenadas geográficas $19^{\circ} 12^{\prime} \mathrm{S}$ e $43^{\circ} 27^{\prime} \mathrm{W}$ e localizada a $1300 \mathrm{~m}$ de altitude. Tem temperatura média anual em torno de $18-20^{\circ} \mathrm{C}$ no verão e abaixo de $15^{\circ} \mathrm{C}$ no inverno. A precipitação média anual varia entre 1450 e $1800 \mathrm{~mm}$. O substrato é do tipo afloramento rochoso, com campo arenoso-pedregoso úmido (Romão, 2003) (Figura 1C).

Em cada fisionomia foram selecionados cinco indivíduos amostrais. De cada indivíduo amostral foi coletada amostra de madeira à altura do peito (1,3m do solo) para os espécimes 

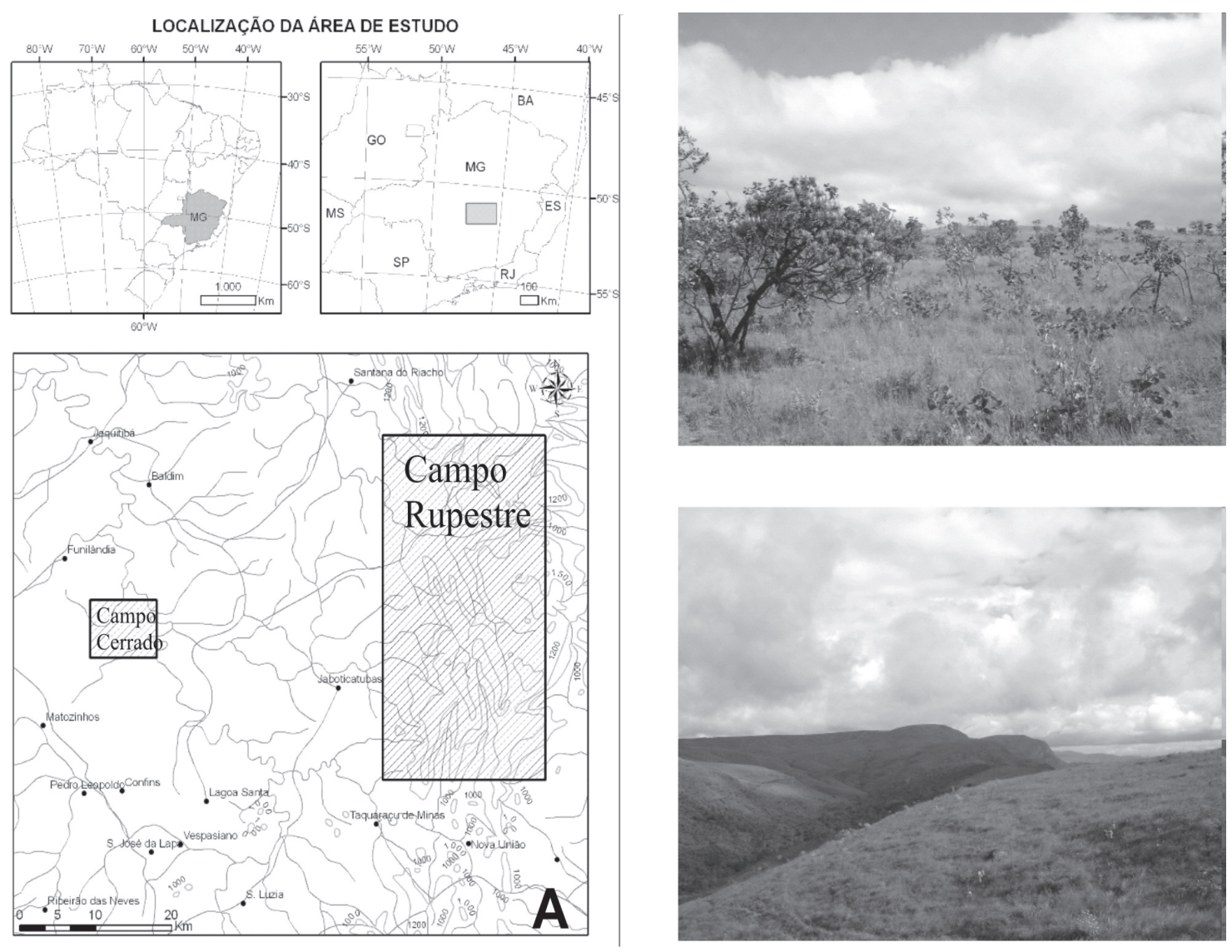

FIGURA 1. Localização e fisionomia das áreas de estudo. A - Campo cerrado no município de Matozinhos/MG, e campo rupestre na Serra do Cipó/MG. B - Campo cerrado. C - Campo Rupestre.

de campo cerrado e na base de um dos perfilhos nos espécimes de campo rupestre. O material coletado foi tombado na Xiloteca JOIw, da Universidade da Região de Joinville (Melo Jr. et al., 2014).

De cada amostra de madeira foram confeccionados dois corpos de prova, um para obtenção de cortes histológicos nos planos anatômicos convencionais em anatomia da madeira (transversal, longitudinal radial e longitudinal tangencial), e o outro para o preparo de material dissociado. Os corpos de prova foram produzidos da porção mais próxima à região cambial, para evitar a juvenilidade do lenho. Para a microtomia e a confecção das lâminas histológicas permanentes, seguiram-se as técnicas apresentadas por Johansen (1940) e Sass (1951). Utilizou-se coloração de azul de Astra e safranina (Bukatsch, 1972) e a montagem foi realizada em verniz vitral (Paiva et al., 2006). Para a análise de elementos celulares dissociados, as amostras de madeira foram estocadas em solução de Franklin (Kraus \& Arduin, 1997) e acondicionadas em estufa a $60^{\circ} \mathrm{C}$ até a completa dissolução da madeira. $\mathrm{O}$ material dissociado foi corado com safranina e montado com a mesma resina sintética. A caracterização anatômica seguiu as orientações e terminologias propostas pelo IAWA Committe (1989). O número de mensurações das características anatômicas do lenho foi fixado em $n=30$.

Para todos os atributos quantitativos estudados foram calculadas médias e desvios-padrão. Para a comparação dos valores médios de cada atributo do lenho entre as populações de $R$. viburnoides de campo cerrado e rupestre utili- 
zou-se o teste t de Student, com $\mathrm{p}<0,05$ em ambiente R (Crawley et al., 2007). Foram calculados os índices de condutividade (IC), dado pela equação de Zimmermann (1983), modificada por Fahn et al. (1986), de mesomorfia (IM) e de vulnerabilidade (IV), seguindo as fórmulas descritas por Carlquist (1977).

\section{RESULTADOS}

A caracterização anatômica de Rudgea viburnoides mostrou diferenças qualitativas entre o lenho dos espécimes das duas fisionomias de cerrado estudadas (Tabela 1), com maior destaque ao agrupamento dos vasos em múltiplos radiais contendo o dobro do número de vasos em campo rupestre. Placas de perfuração simples e reticuladas foram observadas em indivíduos de campo cerrado, em oposição às placas exclusivamente simples em campo rupestre. Pontoações intervasculares guarnecidas foram registradas apenas em campo cerrado. Raios mais largos foram observados em campo cerrado. Cristais prismáticos em células do parênquima radial foram exclusivos em campo rupestre. Nos espécimes de campo cerrado há presença freqüente de tilos.

TABELA 1 - Caracterização e comparação anatômica dos atributos qualitativos do lenho de Rudgea. viburnoides (Rubiaceae), em duas fisionomias de cerrado.

\begin{tabular}{|c|c|c|}
\hline Atributos & Campo Cerrado & Campo Rupestre \\
\hline Camada de crescimento & $\begin{array}{l}\text { distinta, demarcada por } \\
\text { espessamento da parede radial } \\
\text { das fibras }\end{array}$ & $\begin{array}{l}\text { distinta, demarcada por } \\
\text { espessamento da parede radial das } \\
\text { fibras }\end{array}$ \\
\hline \multicolumn{3}{|l|}{ Vasos } \\
\hline porosidade & Difusa & difusa \\
\hline agrupamento & múltiplos 3-6 & múltiplos 3-12 \\
\hline placa de perfuração & simples e raramente reticulada & simples \\
\hline pontoação intervascular & alterna e guarnecida & alterna \\
\hline pontoação raiovascular & $\begin{array}{l}\text { semelhante a intervascular em } \\
\text { forma e tamanho }\end{array}$ & $\begin{array}{l}\text { semelhante a intervascular em } \\
\text { forma e tamanho }\end{array}$ \\
\hline \multicolumn{3}{|l|}{ Fibras } \\
\hline pontoação & Simples & simples \\
\hline septo & Presente & presente \\
\hline espessura da parede & fina a espessa & fina a espessa \\
\hline Parênquima axial & paratraqueal escasso & paratraqueal escasso \\
\hline \multicolumn{3}{|l|}{ Raio } \\
\hline composição & $\begin{array}{l}\text { heterogênio, formado por corpo } \\
\text { procumbente e uma camada } \\
\text { marginal de células eretas ou } \\
\text { quadradas } \\
\text { multisseriado } 2-5 \text {, raramente } \\
\text { unisseriado }\end{array}$ & $\begin{array}{l}\text { heterogênio, formado por corpo } \\
\text { procumbente e uma camada } \\
\text { marginal de células eretas ou } \\
\text { quadradas } \\
\text { multisseriado } 1-2 \text {, raramente } 3 \\
\text { seriado }\end{array}$ \\
\hline \multicolumn{3}{|l|}{$\begin{array}{l}\text { Inclusões minerais e outras } \\
\text { características }\end{array}$} \\
\hline cristais prismáticos & Ausente & em células do parênquima radial \\
\hline \multirow[t]{2}{*}{ tilose } & Presente & ausente \\
\hline & Presente & presente \\
\hline
\end{tabular}


TABELA 2 - Atributos quantitativos avaliados para o lenho de Rudgea viburnoides. Apresentamse valores médios e desvio-padrão (entre parênteses) para cada variável. Valor do teste t de Student e nível de significância (p) para a comparação entre as áreas de estudo (n=30).

\begin{tabular}{lcccc}
\hline \multicolumn{1}{c}{ Atributos } & Campo Cerrado & Campo Rupestre & t & p \\
\hline Elemento de Vaso & & & & \\
diâmetro tangencial $(\mu \mathrm{m})$ & $39.33(5.70)$ & $41.74(3.08)$ & 3.38 & $<0.0008$ \\
comprimento $(\mu \mathrm{m})$ & $68,83(3,96)$ & $41,26(9,07)$ & 8,91 & $<0.001$ \\
freqüência $\left(\mathrm{n}^{\circ} / \mathrm{mm}^{2}\right)$ & $2.04(0.25)$ & $1.54(0.75)$ & 1.54 & $<0.12$ \\
$\begin{array}{l}\text { Fibras } \\
\text { comprimento }(\mu \mathrm{m})\end{array}$ & $133,66(22,30)$ & $65,03(10,08)$ & 15,04 & $<0.0001$ \\
$\begin{array}{l}\text { Parênquima Radial } \\
\text { largura }\left(\mathrm{n}^{\circ} \text { de células) }\right.\end{array}$ & $3.16(0.71)$ & $1.12(0.35)$ & 31.22 & $<0.0001$ \\
altura $\left(\mathrm{n}^{\circ}\right.$ de células) & $9.82(2.46)$ & $9.38(4.59)$ & 1.04 & $<0.2$ \\
largura $(\mu \mathrm{m})$ & $54.41(3.47)$ & $17.00(2.95)$ & 35,00 & $<0.0001$ \\
altura $(\mu \mathrm{m})$ & $441.26(45.57)$ & $360.45(78.50)$ & 3.88 & $<0.0001$ \\
\hline
\end{tabular}

Diferenças estatisticamente significativas foram observadas para os atributos quantitativos do lenho, tais como o diâmetro tangencial de vasos, o comprimento de elemento de vaso, o comprimento de fibras, a largura de raios em número de células, e a largura e altura de raios em $\mu \mathrm{m}$, sendo as maiores médias encontradas nos indivíduos de campo cerrado, exceto para o primeiro atributo mencionado (Tabela 2).

Apesar de não diferir estatisticamente, a frequiência de vasos mostrou-se mais elevada em campo cerrado e inversamente proporcional ao diâmetro tangencial dos vasos. Situação oposta foi obtida para os indivíduos de campo rupestre (Tabela 2). Semelhanças estruturais no lenho foram dadas pelas fibras septadas e pela altura do parênquima radial em número de células. Máculas também foram encontradas nos espécimes de ambas as fisionomias de cerrado.

Maior condutividade hidráulica é atribuída ao lenho de $R$. viburnoides em campo rupestre, que, em contrapartida, acentua o risco ao embolismo e cavitação (Tabela 3). Em ambas as fisionomias, as populações estudadas são consideradas xerófitas, em função dos valores obtidos para o índice de mesomorfia (Tabela 3).

TABELA 3 - Índice de Vulnerabilidade (IV), Índice de Condutividade (IC) e Índice de Mesomorfia (IM) para os dois locais de estudo.

\begin{tabular}{|c|c|c|c|}
\hline Local de estudo & IV (D/F) & IC $\left(\mathbf{r}^{4} / \mathbf{F}\right)$ & IM (IV x C) \\
\hline Campo Rupestre & 27,10 & 123188,19 & 1118,14 \\
\hline Campo Cerrado & 19,27 & 73232,50 & 1326,35 \\
\hline
\end{tabular}

\section{DISCUSSÃO}

A espécie em estudo apresenta madeira com camadas de crescimento distintas, demarcadas por espessamento e achatamento das paredes radiais de fibras, o que representa os inícios e as paradas do crescimento secundário das plantas lenhosas (Zenid et al., 2007). A formação dos anéis de crescimento está relacionada com as condições do ambiente, uma vez que fatores abióticos, como a intensidade luminosa, a temperatura, a disponibilidade hídrica e o espaço para crescimento, estão diretamente relacionados ao crescimento em diâmetro do caule (Savva, 2002). Em regiões tropicais com solos 
bem drenados ou com sazonalidade marcada por períodos de secas e de chuvas, o crescimento das árvores passa por um estado de dormência, levando à formação de anéis anuais de crescimento. Nos ambientes de cerrado, a variação sazonal, efetivamente marcada pela existência de duas estações, uma seca e outra chuvosa, possui maior influência na formação das camadas de crescimento, como o observado em estudo com Copaifera langsdorffii Desf. (Fabaceae) em cerrado stricto sensu (Melo Jr. et al., 2012). A formação de camadas de crescimento é comum na família Rubiaceae e foi observada por Willians \& Léon (2007) e Siegloch et al. (2011).

Vasos de maior diâmetro tangencial foram observados no lenho das plantas de campo rupestre, porém menos numerosos que em campo cerrado. Baas et al. (2004) ressaltam que vasos de maior diâmetro são mais eficientes na condução de água, pois oferecem menor fricção e conduzem maior quantidade de líquido; estes, todavia, são mais propensos à embolia (Sperry et al., 1994). Em razão desse fator, as espécies de ambiente com menor disponibilidade de água tendem a apresentar vasos de menor calibre, enquanto o número de vasos tende a aumentar por unidade de área, para compensar a eficiência no transporte hídrico e evitar embolias (Baas et al., 1983), o que foi observado para a espécie em estudo. Situações opostas ao esperado foram descritas por Ceccantini (1996), que observou vasos de maior diâmetro em Casearia sylvestris Sw. (Flacourticaceae) de campo cerrado, em estudo comparativo da anatomia do lenho em espécies de cerrado e floresta, por Mina-Rodrigues (1986), que encontrou vasos de maior diâmetro no campo cerrado para a espécie Pera glabrata (Schott) Poepp ex. Baill. (Peraceae), e por Marcati et al. (2001), que observaram vasos de maior diâmetro em Copaifera langsdorffii de campo cerrado. No entanto, Baas et al. (1983) afirmam que em ambientes xéricos podem ocorrer vasos de diferentes diâmetros a fim de aumentar a eficiência de condutividade conforme a sazonalidade; assim, em períodos áridos, os vasos mais estreitos são utilizados com maior eficiência e, nos períodos de maior pluviosidade, os vasos mais largos contribuem com a eficiência hidráulica. Outra importante característica referente à condução hidráulica é o comprimento dos elementos de vaso, uma vez que vasos longos transportam água de forma mais segura, ao passo que vasos curtos são mais eficientes na condução (Zimmermann; Milburn 1982). A média do comprimento de elementos de vaso na espécie aqui tratada foi maior em campo cerrado, pois em ambiente com baixa disponibilidade hídrica a espécie investe na condução hidráulica segura, evitando, assim, prejuízos em períodos mais secos. Segundo Metcalfe (1983), quanto maior a altitude e latitude, e menor a disponibilidade hídrica, os vasos vão se tornando mais curtos, estreitos e apresentam-se com menor freqüência.

A presença de placas de perfuração simples, aspecto comum em Rubiaceae, foi observada em 31 espécies desta família por Willians \& León (2011). As placas de perfuração simples podem estar relacionadas à ambientes secos e quentes, sendo mais eficientes no transporte de água e, portanto, mais úteis nos períodos de maior pluviosidade em ambientes áridos (Wheeler; Baas, 1991; Machado et al., 1997). Em campo rupestre, a presença de placa de perfuração foraminada pode estar relacionada com a prevenção a embolias aéreas, através da retenção de bolhas de ar (Wheeler; Baas, 1991).

As pontoações guarnecidas, observadas em indivíduos de campo cerrado, podem estar relacionadas à ambientes secos, conforme observado por Alves e Angyalossy-Alfonso (2000). Esta característica é tida como uma importante adaptação a ambientes sujeitos ao estresse hídrico, tais como os do Cerrado (Machado et al., 1997), por evitar embolias a partir da maior aderência das moléculas de água às paredes das células (Carlquist, 1988). Pontoações guarnecidas também foram observadas com frequiência em várias espécies arbóreas da flora brasileira (Alves; Angyalossy-Alfonso, 2000), em Styrax camporum Pohl. (Styracaceae) de cerrado (Machado et al., 1997) e em Copaifera langsdorffii 
de diferentes fisionomias de cerrado, nos estados de São Paulo e Paraná (Marcati et al., 2001; Melo Jr. et al., 2012).

Fibras mais longas foram observadas em indivíduos de $R$. viburnoides de campo cerrado, cuja arquitetura é caracterizada por arvoretas, diferentemente dos espécimes de campo rupestre, que são arbustos densamente perfilhados. Além de contribuir com o suporte mecânico da planta (Burger; Richter,1991), as fibras desempenham um importante papel na condução de água a longa distância, pois atuam no xilema com o estoque de água por capilaridade nas paredes (Jacobsen et al., 2005). Para Goulart e Marcati (2008), o maior comprimento das fibras no caule pode estar relacionado à função mecânica de suporte da copa das plantas, além de garantir à mesma uma maior flexibilidade deste órgão (Denardi, 2007).

A presença de fibras septadas é característica comum em Rubiaceae (Zevallos Pollito; Tomazello, 2006), e igualmente útil na função de reserva de materiais no xilema (Chalk, 1989).

Um solo pobre em nutrientes, juntamente a um ambiente sazonal, pode favorecer a formação de raios mais largos, que garantiriam o suprimento de nutrientes necessário nos períodos de seca (Goulart; Marcati, 2008). Esta afirmação condiz com o observado no presente estudo, em que raios mais largos foram observados em indivíduos de campo cerrado. Chimelo \& Mattos-filho (1988) também encontraram maiores valores de altura e largura de raios em indivíduos de campo cerrado, comparado aos de floresta mésica. Segundo os autores, esta característica está relacionada às diferenças de umidade, acidez e disponibilidade de nutrientes no solo. Raios mais largos, em ambientes com menor disponibilidade hídrica, foram observados em Eugenia uniflora L. (Myrtaceae) (Marques et al. 2012), Croton urucurana Baill. (Euphorbiaceae) (Luchi, 2004), e Enterolobium contortisiliquum (Vell.) Morong (LeguminosaeMimosoideae) (Lima et al., 2009).

Os cristais prismáticos, registrados nos indivíduos de campo rupestre, podem funcionar como reservas de substância que podem ser incorporadas no ciclo metabólico ou como mecanismo antiherbivoria (Metcalfe \& Chalk, 1989). A maior frequiência de cristais é encontrada em plantas de ambientes secos, como observado por Barajas-Morales (1985), Fahn et al. (1986) e Chimelo \& Mattos-Filho (1988).

A presença de tilos no lenho dos espécimes de campo cerrado constituem obstruções permanentes, resultantes da sucção de células parenquimáticas vizinhas aos vasos; este caráter anatômico pode estar relacionado ao estresse hídrico do ambiente de cerrado, uma vez que a formação de tilos interfere no transporte de água da parte mais interna do caule para a mais externa (Galvão \& Jankowski, 1985).

As máculas, observadas nos indivíduos de ambas as fisionomias de cerrado, são interpretadas como estruturas de origem traumática, relacionadas a danos causados pelo calor ou frio (Carlquist, 1988; Ceccantini, 1996); é o caso da área estudada, que registra geadas anuais.

Segundo Carlquist (1977), plantas com índice de mesomorfia (IM) superior a 200 são consideradas mesomorfas. Os indivíduos de ambos os locais de estudo apresentam IM muito acima de 200, o que significa que estas plantas estão adaptadas a ambientes secos, sendo o maior valor observado para campo cerrado, onde a disponibilidade hídrica é menor. $\mathrm{O}$ índice de vulnerabilidade (IV) indica a propensão de ocorrer embolia, e a conseqüente interrupção do fluxo de água (Carlquist, 1977). Os indivíduos de campo cerrado investem mais na segurança da condutividade, do que os de campo rupestre. $\mathrm{O}$ índice de condutividade (IC), observado em maior valor nos indivíduos de campo cerrado, reflete um ajuste para aumentar a eficiência na quantidade de água transportada, mas também aumenta a propensão de embolias e cavitação (Carlquist, 1977).

A análise comparativa do lenho entre indivíduos procedentes de campo rupestre e campo cerrado revelou ajustes estruturais do lenho, tanto em aspectos qualitativos como quantitativos. As variações anatômicas constatadas sugerem 
que as diferentes condições físico-climáticas das fisionomias de cerrado em estudo podem exercer efeito sobre a plasticidade observada na madeira de Rudgea viburnoides. Tais ajustes mostram a vantajosa funcionalidade ecológica de atributos da madeira que permitem à espécie otimizar seu processo de colonização e sobrevivência em fisionomias de cerrado que exibem diferentes condições na oferta de recursos.

\section{REFERÊNCIAS BIBLIOGRÁFICAS}

ALVES, E.S.; ANGYALOSSY-ALFONSO, V. Ecological trends in the wood anatomy of some Brazilian species. I: growth rings and vessels. IAWA Journal, v. 21, n. 1, p. 3-30, 2000.

ALVES; R.M.S.; STEHMANN, J.R.; ISAIAS, R.M.S.; BRANDÃO, M.G.L. Caracterização botânica e química de Rudgea viburnoides (Cham.) Benth., (Rubiaceae). Revista Brasileira de Farmacognosia, v. 14, n. 1, p. 49-56, 2004.

BAAS, P. The wood anatomical range in Ilex (Aquifoliaceae) and its ecological and phylogenetic significance. Blumea, v. 21, p. 193258, 1973.

BAAS, P.; WERKER, E.; FAHN, A. Some ecological trends in vessel characters. IAWA Bulletin, v. 4, n. 2-3, p. 2-3, 1983.

BARAJAS-MORALES, J. Wood structural difference between trees of the tropical forests in México. IAWA Bulletin, v. 6, n. 4, p. 355-364, 1985.

BAAS, P.; EWERS, F.W.; DAVIS, S.D.; WHEELER, E.A. Evolution of xylem physiology. In: POOLE, I. \& HEMSLEY, A., editors. Evolution of plant physiology. London: Elsevier Academic Press, 2004. p. 273-295.

BARROS, C.F.; MARCON-FERREIRA, M.L.; CALLADO, C.H.; LIMA, H.R.P.; CUNHA, A.M.; MARQUETE, O.; COSTA, C.G. Tendências ecológicas na anatomia da madeira de espécies da comunidade arbórea da Reserva Biológica de Poço das Antas, Rio de Janeiro, Brasil. Rodriguésia, Rio de Janeiro, v. 57, n. 3, p. 443- 460, 2006.

BRADSHAW, A.D. Unravelling phenotypic plasticity - why should we bother? New Phytol., v. 170, n. 4, p. 644-648, 2006

BUKATSCH, F. Bemerkungen zur doppelfärbung astrablau-safranin. Mikrokosmos, v. 61, n. 8, p. 33-36, 1972.
BURGER, L.M; RICHTER, H.G. Anatomia de madeira. Nobel, 1991. 154p.

CARLQUIST, S. Ecological strategies in xylem evolution. Berkeley and Los Angeles: University of California Press, 1975. 259p.

CARLQUIST, S. Ecological factors in wood evolutions: a floristic approach. American Botany, v. 64, n. 7, p. 887-896, 1977.

CARLQUIST, S. Comparative wood anatomy systematic ecological and evolutionary aspects of Dicotiledonean wood. Springer Verlag, 1988. 460p.

CECCANTINI, G. Anatomia ecológica do lenho em espécies de cerrado e mata: Casearia sylvestris Sw. e Machaerium villosum Vog. 1996. 117 p. Dissertação de Mestrado. Universidade de São Paulo, São Paulo.

CHALK, L. Fibres. In: METCALFE, C.R.; CHALK, L. Anatomy of the Dicotyledons. Wood structure and conclusion of the general introduction. Oxford University Press. 1989. p. 28-38.

CHIMELO, J.P.; MATTOS-FILHO, A. Observações preliminares na estrutura da madeira de cinco espécies de folhosas de diferentes locais do Brasil. IUFRO, 1988. 117p.

CRAWLEY M.J. The R book. John Wiley and Sons, 2007. 951p.

DENARDI, L. Anatomia e flexibilidade do caule de quatro espécies lenhosas para o manejo biotécnico de cursos de água. Santa Maria: Programa de Pós-Graduação em Engenharia Florestal, 2007. Tese (Doutorado em Engenharia Florestal - UFSM). 111p.

DURIGAN, G.; BAITELLO, J.B.; FRANCO, G.A.D.C.; SIQUEIRA, M.F.D.E. Rudgea viburnoides (Cham.) Benth. Plantas do Cerrado Paulista: Imagens de uma paisagem ameaçada. Páginas e letras, 2004. 474p.

EMBRAPA. Sistema brasileiro de classificação de solos. 3. ed. Brasília: Embrapa; 2013. 342p.

FAHN, A.; WERKER, E.; BAAS, P. Wood anatomy and identification of trees and shrubs from the Israel and adjacent regions. The Israel Academy of Sciences of Humanities, 1986, 221p.

GALVÃO, A.P.M.; JANKOWSKY, I.P. Secagem Racional da Madeira. Nobel, 1985. 111p.

GOULART, S.L.; MARCATI, C.R. Anatomia comparada do lenho em raiz e caule de Lippia salviifolia Cham. (Verbenaceae). Revista Brasileira de Botânica, v. 31, n. 2, p. 263-275, 2008.

IAWA COMMITTEE. List of microscopic features for hardwood identification. IAWA Bulletin, v. 10, n. 3 , p. 220-332, 1989. 
JACOBSEN, A.L.; EWERS, F.W.; PRATT, R.B.; PADOCK III, E.A.; DAVIS S.D. Do xylem fibers affect vessel cavitation resistance? Plant physiology, v. 135, n. 1, p. 546-556, 2005.

JOHANSEN, D. A. Plant microtechnique. Mac Graw-Hill, 1940. 523p.

KLINK, C.; MACHADO, R.A. Conservação do Cerrado brasileiro. Megadiversidade, Belo Horizonte, v. 1, n. 1, p. 147-155, 2005.

KOEK-NOORMAN, J. The wood anatomy of Gardenieae, Ixoreae and Mussaendeae (Rubiaceae). Acta Botanica Neerladica, v. 21, n. 3, p. 301- 320, 1972.

KOEK-NOORMAN, J.; HOGEWEG, P. The wood anatomy of Vanguerieae, Cinchoneae, Condamineae, and Rondeletieae (Rubiaceae). Acta Botanica Neerlandica, v. 23, n. 5-6, p. 627653, 1974.

KRAUS, J.E.; ARDUIN, M. Manual básico de métodos em morfologia vegetal. Seropédica, 1997. $198 \mathrm{p}$.

LIMA, R.S.; OLIVEIRA, P.L.; RODRIGUES L.R. Anatomia do lenho de Enterolobium contortisiliquum (Vell.) Morong (Leguminosae-Mimosoideae) ocorrente em dois ambientes. Revista Brasileira de Botânica, v. 32, n. 2, p. 361-374, 2009.

LUCHI, A.E. Anatomia do lenho de Croton urucurana Baill. (Euphorbiaceae) de solos com diferentes níveis de umidade. Revista Brasileira de Botânica, v. 27, n. 2, p. 271-280, 2004.

MACHADO, S.R.V.; ANGYALOSSY-ALFONSO, V.; MORRETES, B.L. Comparative wood anatomy of root and stem in Styrax camporum (Styracaceae). IAWA Bulletin, v. 18, n. 1, p. 1325, 1997.

MACHADO, S.R.; RODELLA, R.A.; ANGYALOSSY, V; MARCATI, C.R. Structural variations in root and stem wood of Styrax (Styracaceae) from Brazilian forest and cerrado. IAWA Journal, v. 28, n. 2, p. 173-188, 2007.

MARCATI, C.R.; ANGYALOSSY-ALFONSO, V.; BENETATI, L. Anatomia comparada do lenho de Copaifera langsdorffii Desf. (LeguminosaeCaesalpinoideae) de floresta e cerradão. Revista brasileira de Botânica, v. 24, n. 3, p. 311-320, 2001.

MARQUES, P.A.; CALLADO, K.L.; BARROS, C.F.; COSTA, C.G. Variação Intraespecífica do lenho de Eugenia uniflora L. em duas diferentes fisionomias do complexo vegetacional Atlântico. Floresta e Ambiente, v. 19, n. 4, p. 483-496, 2012.
MELO Jr.; J.C.F.; BONA, C.; CECCANTINI, G. Anatomia foliar de Copaifera langsdorffii Desf. (Leguminosae): interpretações ecológicas em diferentes condições edáficas de Cerrado. Biotemas, v. 25, n. 4, p. 29-36, 2012.

MELO Jr., J.C.F.; AMORIM, W.A.; SILVEIRA, E.R. A xiloteca (coleção Joinvillea ? JOIw) da Universidade da Região de Joinville. Rodriguésia, v. 65, n. 4, p. 1057-1060, 2014.

METCALFE, C.R. Anatomy, phylogeny and taxonomy. In: METCALFE, C.R.; CHALK, L. Anatomy of Dicotyledons. Oxford University Press, 1983, p. 98-125.

METCALFE, C.R.; CHALCK, L. Anatomy of the Dicotyledons. Oxford: Oxford Science Publications, 1989. 297p.

MINA-RODRIGUES, E.M.C. Estudo morfoanatômico dos órgãos vegetativos de Pera glabrata Baill. (Euphorbiaceae), em material procedente de mata mesófila semidecídua e de cerrado. Rio Claro: Universidade Estadual Paulista, 1986. 103 p. Dissertação de Mestrado.

PATEL C.R.; PATEL D.A. Occurrence and significance of cell inclusions in Rubiaceae. Current Botany, v. 2, n. 4, p. 6-11, 2011

PAIVA, J.G.A.; FANK-DE-CARVALHO, S.M.; MAGALHÃES, M.P.; RIBEIRO, D.G. Verniz vitral incolor 500Ò: uma alternativa de meio de montagem economicamente viável. Acta Botânica Brasileira, v. 20, n. 2, p. 257-264, 2006.

ROGERS, G.K. The genera of Rubiaceae in the Southeastern United States, part II. Subfamily Rubioideae, and subfamily Cinchonoideae revisited (Chiococca, Erithalis, and Guettarda). Harvard Papers in Botany, v.10, n. 1, p. 1-45, 2005.

ROMÃO, G.O. Flora da Serra do Cipó: Ericaceae. Universidade de São Paulo: São Paulo, 2003. Dissertação de Mestrado. 147 p.

SASS, J.E. Botanical microtechnique. 2 ed. State College Press, 1951. 228p.

SAVVA, Y.; SCHWEINGRUBER, F.; MILYUTIN, L.; VAGANOV, E. Genetic and environmental signals in tree rings from different provenances of Pinus sylvestris L. planted in the southern taiga, central Siberia. Trees, v. 16, n. 4, p. 313324, 2002.

SHEPHERD, G.J. Conhecimento e diversidade de plantas terrestres do Brasil. Secretaria de Biodiversidade e Florestas, Ministério do Meio Ambiente, 2000. 53p. 
SIEGLOCH, A.M.; MARCHIORI, J.N.C.; SANTOS, S.R. Anatomia do lenho de Cephalanthus glabratus (Spreng.) K. Schum. (Rubiaceae). Balduinia, Santa Maria, v. 11, n. 31, p. 20-26, 2011.

SPERRY J.S.; NICHOLS K.L.; SULLIVAN J.E.; EASTLACK S.E. Xylem embolism in ringporous, diffuse-porous, and coniferous trees of northern Utah and interior Alaska. Ecology, v. 75, n. 6, p. 1736-1752, 1994.

SONSIN, J.O.; GASSON, P.E.; MACHADO, S.R.; CAUM, C.; MARCATI, C.R. Atlas da Diversidade de Madeiras do Cerrado Paulista. Botucatu: FEPAF, 2014. 423p.

VOIGT, A.A.; SOFFIATTI, P.; TOMAZELLO, M.; LISI, C.; BOEGER, M.R.T. Anatomia comparada do lenho de Caryocar brasiliense Camb. (Caryocaraceae) em fisionomias de cerradão e cerrado sensu stricto. Rodriguésia, v. 61, n. 4, p. 603-613, 2010.

WILLIANS, J.; LEÓN, H. Anatomía de la madera de 31 especies de Rubiaceae en Venezuela. Acta Botánica Venezuelica, v.34, n. 2, p. 347-379, 2011.
WHEELER, E.A.; BAAS, P. A survey of the fossil record for dicotyledonous wood and its significance for evolutionary and ecological wood anatomy. IAWA Bulletin, v. 12, n. 5, p. $272-$ 332, 1991.

ZEVALLOS POLLITO, P.A.; TOMAZELLO, M. Anatomia do lenho de Uncaria guianensis e $U$. tomentosa (Rubiaceae) do estado do Acre, Brasil. Acta amazônica, v. 36, n. 2, p. 69-176, 2006.

ZENID, G.J. Madeiras e suas características. In: OLIVEIRA, J.T.S., FIEDLER, N.C.; NOGUEIRA, M. (Org.). Tecnologias aplicadas ao setor madeireiro. Suprema Gráfica e Editora, 2007. p. 125-158.

ZIMMERMANN, M.H. Xylem structure and the ascent of sap. Spinger-Verlag, 1983, 163p.

ZIMMERMANN, M.H.; MILBURN J.A. 1982. Transport and storage of water. In: LANGE, O.L.; NOBEL, P.S.; OSMOND, C.B.; ZIEGLER. H. Physiological Plant Ecology: Water relations and carbon assimilation. New York, p. 135-152. 